\section{A case of acute promyelocytic leukemia with morphologic multilineage dysplastic changes}

\author{
Setsuki Isono, ${ }^{1}$ Katsuyasu Saigo, ${ }^{2}$ \\ Keiko Nagata, ${ }^{1}$ Keiko Numata, ${ }^{1}$ \\ Toshiaki Kojitani, ${ }^{1}$ Akiharu Okamura, ${ }^{3}$ \\ Akihiko Nishizawa, ${ }^{4}$ Masafumi Takata, ${ }^{2}$ \\ Mariko Takenokuchi, ${ }^{4}$ Eiji Tatsumi ${ }^{5}$ \\ ${ }^{1}$ Clinical Laboratory, Kakogawa West City \\ Hospital; ${ }^{2}$ Faculty of Pharmacological \\ Sciences, Himeji Dokkyo University; \\ ${ }^{3}$ Section of Pathology, Kakogawa West \\ City Hospital; ${ }^{4}$ Section of Internal \\ Medicine, Kakogawa West City Hospital; \\ ${ }^{5}$ Department of Nutrition and Health, \\ Sagami Women's University, Japan
}

\section{Abstract}

Although reports of typical acute promyelocytic leukemia (APL) cases rarely mention dysplastic changes, this report concerns a rare case of APL with tri-lineage dysplastic changes resembling the characteristic features of myelodysplastic syndrome (MDS). The patient, a 77-year-old Japanese male, was diagnosed as having pancytopenia with hematologic morphological abnormalities comprising micromegakaryocytes, neutrophils with hypo-granulation and negative peroxidase activity, and erythroblasts containing nuclei with abnormalities such as karyorrhexis. Although there is one report of a case of transformation of de novo MDS into APL and several reports of cases of therapy-related MDS transformed into APL, our patient had no history of cytopenia or of either chemo or radiation therapy. Our case can thus be considered to constitute a rare case of APL with dysplastic morphology.

\section{Introduction}

Myelodysplastic syndrome (MDS) frequently transforms into acute myelogenous leukemia, but rarely into acute promylocytic leukemia (APL). ${ }^{1}$ We experienced a rare case of elderly APL featuring various morphological abnormalities resembling characteristics of MDS, although the patient did not go through a stage perceptibly complicated with any form of cytopenia before the diagnosis of APL.

\section{Case Report}

A 77-year-old male was suffering from low- grade fever and general fatigability in April 2011. After the possibility of pancytopenia was pointed out by a general physician, he was referred to Kakogawa West City Hospital, Japan. A chest X-ray indicated the presence of pneumonia and yielded the following laboratory findings: leukocyte count, $0.84 \times 10^{9} / \mathrm{L}$; red blood cell count, $2.75 \times 10^{12} / \mathrm{L}$; hemoglobin value, $8.5 \mathrm{~g} / \mathrm{dL}$; hematocrit value, $26.5 \%$; platelet count, $55 \times 10^{9} / \mathrm{L}$; prothrombin time, $13.8 \mathrm{sec}$; fibrinogen level, $372 \mathrm{mg} / \mathrm{dL}$ and fibrin degradation product (FDP) level, 88.7 micro $\mathrm{g} / \mathrm{mL}$. These findings pointed to the presence of pancytopenia and a hyperfibrinolytic state.

Differential leukocyte counts were $10 \%$ for neutrophils, $83 \%$ for lymphocytes and $1 \%$ for basophils. Morphological examination detected frequent hypo-granular and peroxidase (POX)-negative neutrophils (Figure 1A and B).

Blood chemistry test findings were: CRP, $20.45 \mathrm{mg} / \mathrm{dL} ;$ AST, 20 IU/L; ALT, 8 IU/L; LD, 175 IU/L; ALP, 238 IU/L; BUN, 55 mg/dL; creatinine, $2.74 \mathrm{mg} / \mathrm{dL}$ and uric acid, $8.7 \mathrm{mg} / \mathrm{dL}$, indicating severe inflammation due to pneumonia and renal dysfunction.

Iliac bone marrow aspiration produced a total nucleated cell count of $59 \times 10^{9} / \mathrm{L}$ and a megakaryocyte count of $25 \times 10^{6} / \mathrm{L}$, while the differential count showed the bone marrow consisted of $28.2 \%$ myeloblasts and $55.2 \%$ abnormal promyelocytes, frequently containing Auer rods or faggot cells (Figure 2A and B). These cells were strongly positive for POX staining (Figure 2C). Micromegakaryocytes and megakryocytes with non-lobulated nuclei or isolated multi-nuclei (Figure 3A-D) as well as erythroblasts showing karyorrhexis or nuclei with abnormal configuration (Figure $3 \mathrm{E}$ and $\mathrm{F}$ ) were also detected. Surface marker analysis produced positive results for CD13 (88.9\%), CD33 (98.7\%), CD64 (70.8\%), CD65 (42.9\%), CD71 (51.8\%), CD117 (84.8\%) and negative results for CD2, CD7, CD19, CD14, CD15, CD11b, CD34, HLA-DR, and gycophorinA. RT-PCR detected the presence of a PMLRARA chimeric mRNA of $2.0 \times 10^{4}$ copies/micro g RNA, and chromosome analysis showed $46 \mathrm{XY}, \mathrm{t}(15 ; 17)(\mathrm{q} 22 ; \mathrm{q} 12)$ in $25 \%$ of the cells analyzed. These results indicated the patient had APL with MDS-like morphological abnormalities complicated with disseminated intravascular coagulation (DIC), pneumonia and renal insufficiency.

Although treatment with all-trans-retinoic acid (ATRA) combined with antibiotics therapy was started immediately, renal insufficiency deteriorated and the patient died on the 14th day after admission. The renal insufficiency was considered to be result of pre-existing dysfunction and adverse effects of antibiotics or anti-inflammatory drugs, although the possibility of differentiation syndrome could not be completely excluded. After the initiation of ATRA administration, the leukocyte counts
Correspondence: Katasuyasu Saigo, Faculty of Pharmacological Sciences, Himeji Dokkyo University, 7, Kami-ohno, Himeji, Hyogo 6708524, Japan.

Tel. +81.79.2236844 - Fax: +81.79 .2236853 .

E-mail: ksaigo@himeji-du.ac.jp

Contributions: SI, A0, concept and design, data acquisition and interpretation; KS, concept and design, data interpretation, drafting of article; MTake, data interpretation, drafting of article; ET, concept and design, data interpretation; KNa, $\mathrm{KNu}$, TK, data acquisition; MTaka, AN, data interpretation.

Conflict of interests: the authors declare no conflict of interests.

Key words: acute promyelocytic leukemia, dysplasia, all-trans retinoic acid.

Received for publication: 19 September 2012. Revision received: 27 November 2012. Accepted for publication: 7 January 2013.

This work is licensed under a Creative Commons Attribution NonCommercial 3.0 License (CC BYNC 3.0).

(C) Copyright S. Isono et al., 2013

Licensee PAGEPress, Italy

Hematology Reports 2013; 5:e5

doi:10.4081/hr.2013.e5

increased from $1 \times 10^{9} / \mathrm{L}$ to $5.7 \times 10^{9} / \mathrm{L}$ and the neutrophil percentage from $21 \%$ to $62 \%$. Because mature neutrophils with Auer rods were observed in peripheral blood (Figure 1C), the patient's leukemia was considered to be responsive to ATRA treatment.

\section{Discussion and Conclusions}

Initially, our patient was thought to have MDS complicated with pneumonia and DIC, but the laboratory findings disclosed he was suffering from APL. There are three possible explanations for the morphological abnormalities resembling the characteristic features of MDS. First, this was possibly a rare case of MDS transformed into APL. Ogawa et al. reported a case of RAEB in transformation with $15 \% \mathrm{t}(15 ; 17),{ }^{2}$ which changed to APL after several months and is the only case report of de novo MDS transformed into APL. On the other hand, there are no reports of even APL cases with chromosome 7 abnormalities that possess morphological abnormalities. ${ }^{3}$ Although Varma et al. reported that variant forms of APL cases frequently express myelodysplastic abnormalities, our patient was a typical APL. ${ }^{4}$ However, the diagnosis of MDS 
A

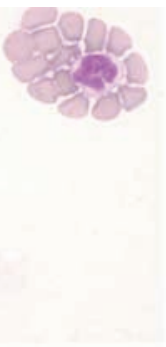

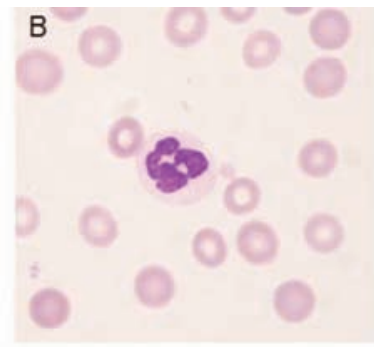

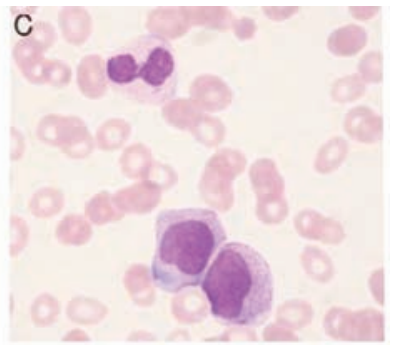

Figure 1. Peripheral blood leukocytes. A) peroxidase (POX) negative neutrophil; B) hypogranular neutrophil (May-Giemsa staining); C) neutrophil containing Auer body after initiation of ATRA treatment (May-Giemsa staining).
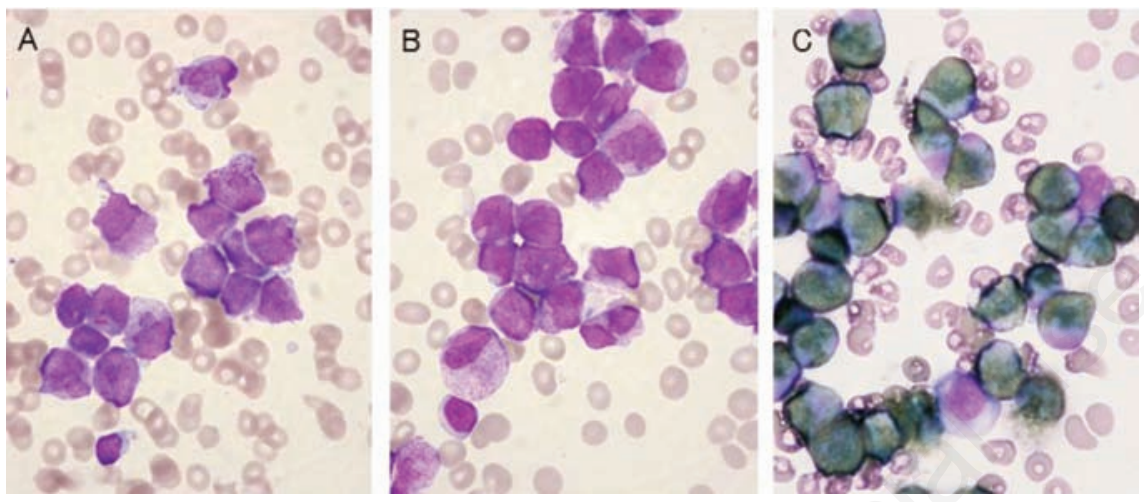

Figure 2. Typical acute promyelocytic leukemia (APL) cells in bone marrow. A) and B) APL cells with hypergranular cytoplasm and Auer body or faggot cells (May-Giemsa staining); C) strongly positive staining for peroxidase.
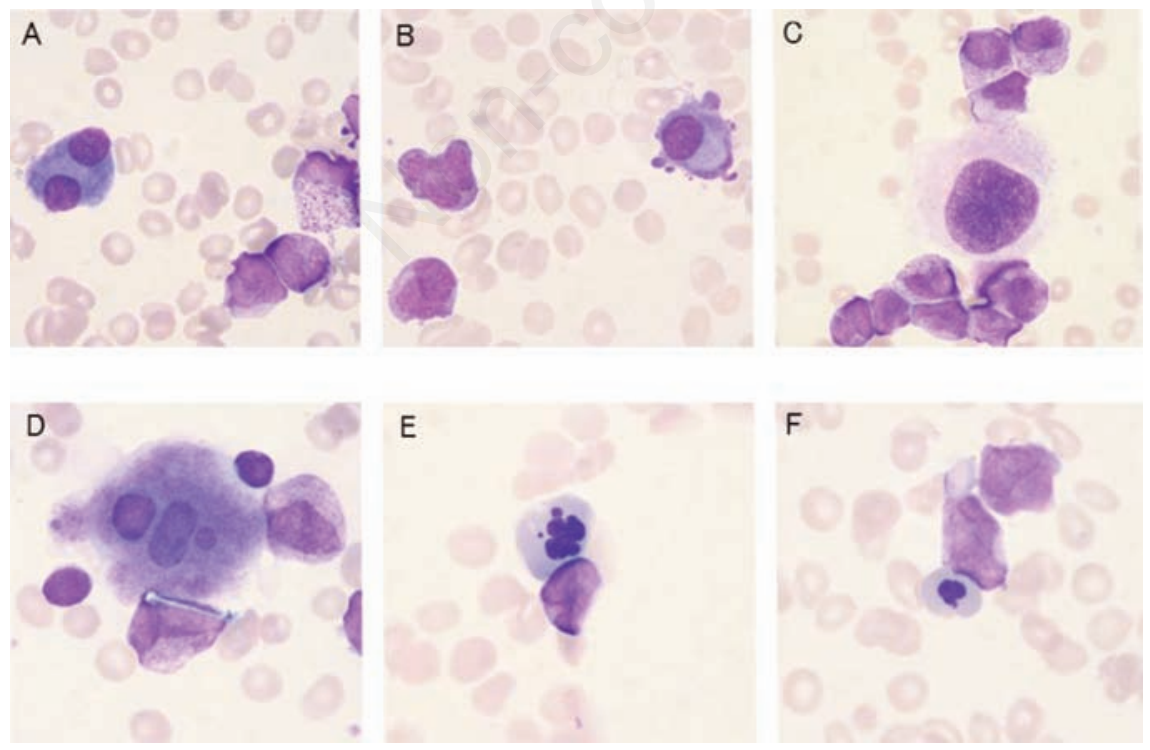

Figure 3. Atypical cells in bone marrow smear (May-Giemsa staining). A) and B) micro megakaryocytes; C) megakryocyte with non-lobulated nucleus; D) megakaryocyte with isolated multi-nuclei; E) erythroblast showing karyorrhexis; F) erythroblast with a nucleus showing abnormal configuration. could not be confirmed in our case because there was no past history of cytopenia.

There are several reports of cases of therapy-related MDS transformed into APL. Anderson et al. reported three such cases among 41 secondary APL cases. ${ }^{5}$ Furthermore, Wolach et al. reported a case responsive to treatment with ATRA and arsenic trioxide that developed APL from therapy-related MDS over the course of several years. ${ }^{6}$ However, our patient had no history of chemotherapy or irradiation.

The third possibility is that, according to the WHO classification of tumors of hematopoietic and lymphoid tissues, our patient could have been classified as acute myelogenous leukemia (AML) with myelodysplasia-related changes. ${ }^{7}$ Although this specific category excludes cases characterized by genetic abnormalities of AML accompanied by recurrent abnormalities such as $\mathrm{t}(15 ; 17)$, our case might be classified in this category.

According to Haferlach et al., morphologic dysplasia in de novo AML reportedly does not correlate with prognosis. ${ }^{8}$ Although our patient also seemed to respond to ATRA, he died from complicating renal insufficiency and infection.

To summarize, we encountered an elderly case with APL associated with dysplastic changes resembling myelodysplastic syndrome.

\section{References}

1. de Souza Fernandez T, Ornellas MH, Otero de Carvalho LD, et al. Chromosomal alterations associated with evolution from myelodysplastic syndrome to acute myeloid leukemia. Leuk Res 2000;24:83948.

2. Ogawa K, Shineha H, Abe R, et al. Acute promyelocytic leukemia with a history of RAEB in transformation and the 15/17 translocation. Rinsho Ketsueki 1989;30: 67-71. [Article in Japanese].

3. Vial JP, Mahon FX, Pigneux A, et al. Derivative (7)t(7;8)(q34;q21): a new additional cytogenetic abnormality in acute promyelocytic leukemia. Cancer Genet Cytogenet 2003;140:78-81.

4. Varma N, Dash S, Sarode R. Correlation between morphological and cytochemical heterogeneity of acute promyelocytic leukemia (APL) and its association with myelodysplasia. Indian J Cancer 1990;27: 166-71.

5. Anderson MK, Larson RA, Mauritzson N, et al. Balanced chromosome abnormalities inv(16) and $t(15 ; 17)$ in therapy-related myelodysplastic syndromes and acute leukemia: Report from an international workshop. Genes Chromosomes Cancer 
2002;33:395-400.

6. Wolach 0, Yeshurun M, Amariglio N, et al. Acute promyelocytic leukemia with a smoldering course associated with therapyrelated myelodysplastic syndrome. Acta Haematol 2011;126;152-6.

7. Arber DA, Brunning RD, Orazi, Bain BJ. Acute myeloid leukemia with myelodys- plastia-related changes. In: Swerdlow SH, Campo E, Harris NL, et al, eds. WHO classification of tumors of haematopoietic and lymphoid tissues, 4th ed. Lyon, France: International agency for research on cancer (IARC); 2008. pp.124-126.

8. Haferlach T, Schoch C, Loffler H, et al. Morphologic dysplasia in de novo acute myeloid leukemia (AML) is related to unfavorable cytogenetics but has no independent prognostic relevance under the conditions of intensive induction therapy: results of a multicenter analysis from the German AML cooperative group studies. J Clin Oncol 2003;21:256-65. 